\title{
Clinical utility of neurostimulation devices in the treatment of overactive bladder: current perspectives
}

This article was published in the following Dove Press journal:

Medical Devices: Evidence and Research

I June 2017

Number of times this article has been viewed

\author{
Dick AW Janssen' \\ Frank MJ Martens' \\ Liesbeth $L$ de Wall' \\ Hendrikje MK van Breda ${ }^{2}$ \\ John PFA Heesakkers'
}

'Department of Urology, Radboud University Nijmegen Medical Center, Nijmegen, ${ }^{2}$ Department of Urology, University Medical Center Utrecht, Utrecht, the Netherlands
Correspondence: Dick AW Janssen Department of Urology, Radboud University Nijmegen Medical Center, Geert Grootteplein 10, 6500 HB, Nijmegen, the Netherlands Email dick.janssen@radboudumc.nl
Objectives: This review describes the evidence from established and experimental therapies that use electrical nerve stimulation to treat lower urinary tract dysfunction.

Methods: Clinical studies on established treatments such as percutaneous posterior tibial nerve stimulation (P-PTNS), transcutaneous electrical nerve stimulation (TENS), sacral nerve stimulation (SNS) and sacral anterior root stimulation (SARS) are evaluated. In addition, clinical evidence from experimental therapies such as dorsal genital nerve (DGN) stimulation, pudendal nerve stimulation, magnetic nerve stimulation and ankle implants for tibial nerve stimulation are evaluated.

Results: SNS and P-PTNS have been investigated with high-quality studies that have shown proven efficacy for the treatment for overactive bladder (OAB). SARS has proven evidencebased efficacy in spinal cord patients and increases the quality of life. TENS seems inferior to other OAB treatments such as SNS and P-PTNS but is noninvasive and applicable for ambulant therapy. Results from studies on experimental therapies such as pudendal nerve stimulation seem promising but need larger study cohorts to prove efficacy.

Conclusion: Neurostimulation therapies have proven efficacy for bladder dysfunction in patients who are refractory to other therapies.

Significance: Refinement of neurostimulation therapies is possible. The aim should be to make the treatments less invasive, more durable and more effective for the treatment of lower urinary tract dysfunction.

Keywords: neuromodulation, overactive bladder syndrome, sacral nerve stimulation, sacral anterior root stimulation, PTNS, implant

\section{Introduction}

The urinary bladder is a low-pressure storage reservoir for urine. Neurological control of the bladder, urethra and pelvic floor muscles is complex and depends on different peripheral, spinal and central nerves and multiple reflex pathways. ${ }^{1}$ Learning to adequately control the bladder reflexes is a difficult task in human development and takes an average of 5 years. ${ }^{2,3}$ The lower urinary tract is sensitive for disturbances. Overactive bladder $(\mathrm{OAB})$ is a complex of storage symptoms that includes urgency with or without urinary incontinence, frequency and nocturia. Idiopathic OAB (iOAB), the most common form, has an unresolved etiology, but aging is an important risk factor. ${ }^{4}$ The following four factors are believed to play a role in OAB etiology: 1) enhanced afferent nerve activation, 2) increased efferent excitation, 3) decreased inhibition by the central nervous system (CNS) and 4) phasic smooth muscle contractions. ${ }^{4}$ 
Bladder control can also be one of the first mechanisms to fail in neurodegenerative diseases. ${ }^{5}$ There are many neurological disorders that can result in lower urinary tract dysfunction. Some examples of these are multiple sclerosis, Parkinson's disease and spinal cord injury (SCI). In the bladder, this often causes OAB. Contrastingly, this can also cause detrusor hypocontractility and the inability to void adequately, which is frequently seen in cauda syndrome and spina bifida patients. Finally, neurologic disturbances can cause obstructive voiding due to detrusor external sphincter dyssynergia (DESD), which is a flawed coordination between bladder and external urethral sphincter (EUS). ${ }^{3}$ A combination between severe detrusor overactivity (DO) and DESD can cause renal reflux and insufficient drainage of the upper urinary tract. This results in high pressures that can cause kidney damage.

Lower urinary tract dysfunction has a profound negative impact on the quality of life. ${ }^{6}$ For decades now, urologists have designed and implemented neurostimulation therapies to treat lower urinary tract dysfunction with success. Many of these different therapies have an unique approach and are clinically applied worldwide (Figure 1). This review outlines the current knowledge on neurologic control and dysfunction of the lower urinary tract and describes how the different neurostimulation therapies intervene in these processes. Clinical evidence is reviewed to highlight the advantages and disadvantages of different neurostimulation techniques.
A

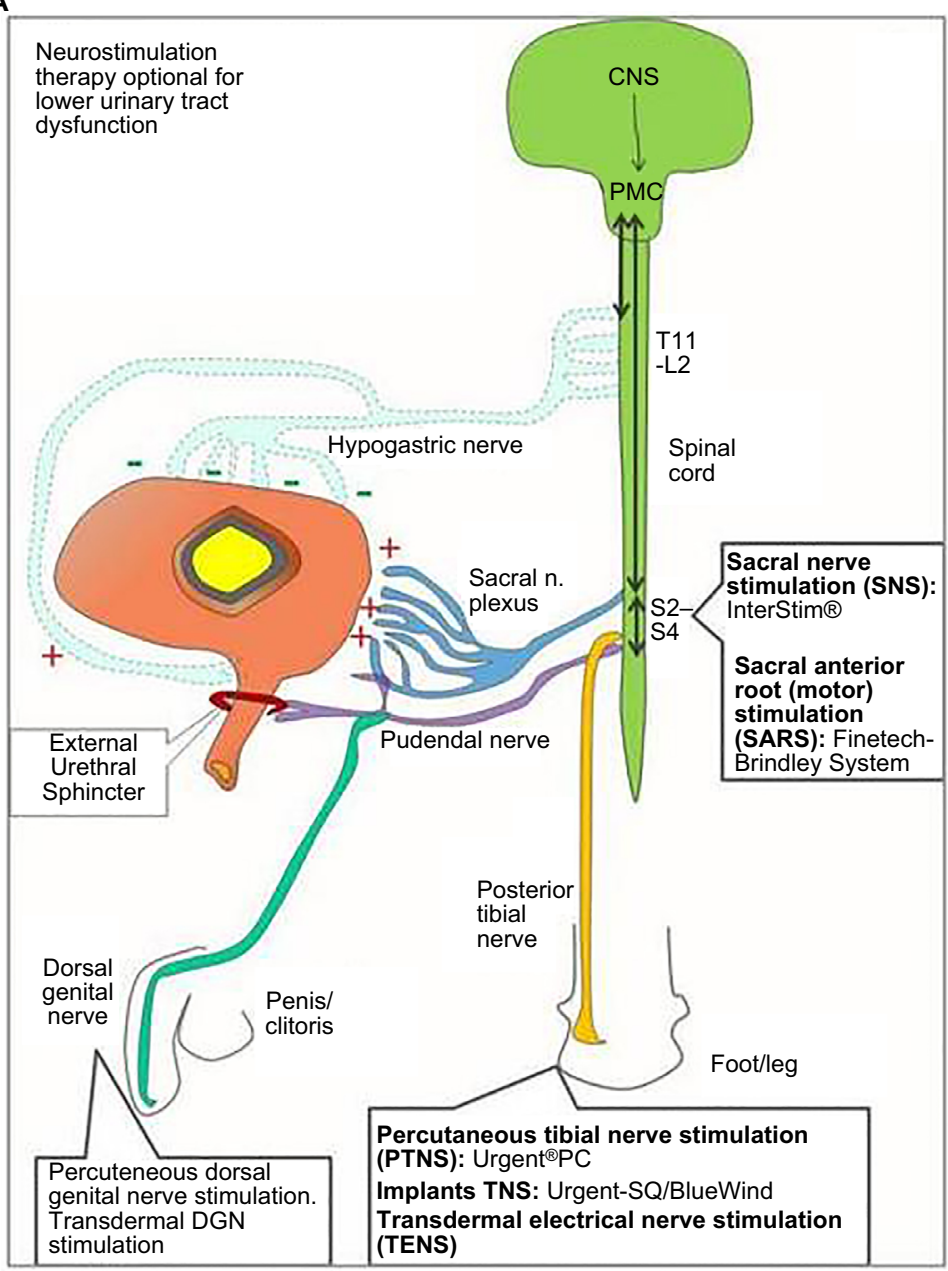

B

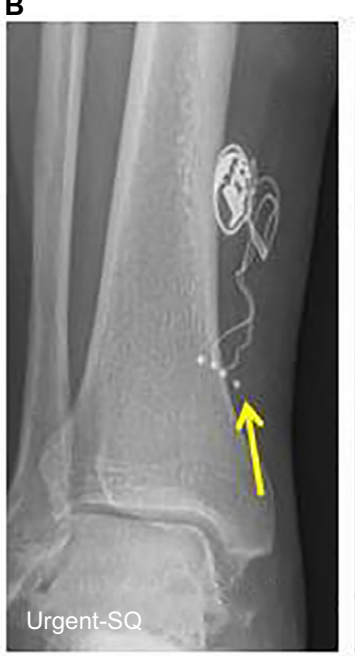

C

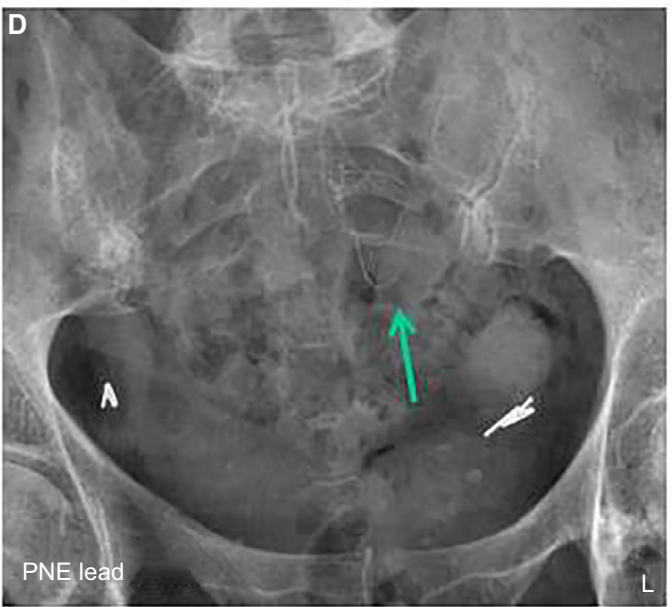

Figure I Summary of therapeutic options (clinical and experimental) for electrical nerve stimulation for lower urinary tract dysfunction.

Notes: (A) Different sites where electrical nerve stimulation is applied for the treatment of lower urinary tract dysfunction. (B) An X-ray image (AP) of the right ankle area of a patient who has an Urgent-SQ ${ }^{\mathrm{TM}}$ implantable device for on demand posterior tibial nerve stimulation. The yellow arrow depicts the wire electrode fixation near the posterior tibial nerve. (C) An X-ray image (lateral) of a right ankle of a patient with a BlueWind ${ }^{\circledR}$ implantable device for on demand posterior tibial nerve stimulation. The electrodes are positioned on the upper and lower sides of the device (green arrows). (D) An X-ray (AP) of the pelvic area from a patient undergoing the PNE test phase for evaluation if SNS is effective. The green arrow is the percutaneously placed wire electrode that is inserted through the left S3 foramen. If effective, the patient will receive a permanent tined lead electrode and in a later phase an InterStim implant.

Abbreviations: AP, anterior posterior view; CNS, central nervous system; DGN, dorsal genital nerve; PNE, percutaneous nerve evaluation; SNS, sacral nerve stimulation; PMC, pontine micturition center. 


\section{Lower urinary tract control}

The lower urinary tract is semiautonomous. This means that one cannot influence the force of a bladder contraction, but it is possible, to a large degree, to suppress bladder contractions during the storage phase, initiate voiding and to consciously control the EUS. For successful storage and voiding, there has to be a finely tuned coordination between the detrusor smooth muscles and the bladder outlet, which consist of the bladder neck (internal sphincter) and EUS. Besides this, there also has to be adequate sensory signaling to monitor bladder filling. The efferent and afferent pathways that control micturition are organized with at least three different peripheral nerves that innervate the bladder and sphincters, which include the parasympathic, sympathic and somatic nerves, and each will be described in detail in the following paragraphs.

Bladder detrusor contraction is controlled by the parasympathic sacral nerves (pelvic nerve) that originate from the S2-S4 region in the spinal cord ${ }^{1,7}$ (Figure 2). The sacral nerves hold besides efferent fibers, many afferent fibers that convey bladder-filling sensations. The sacral nerves use the neurotransmitter acetylcholine (ACh) and muscarinic receptors (mostly M3, also M2) for signaling. These muscarinic receptors are the main pharmacological target sites to treat OAB (Figure 2). Examples of this are widespread use of antimuscarinics and intradetrusor botulinum toxin
A injections that are both evidence-based therapies that are included in all major therapy guidelines for neurogenic $\mathrm{OAB}$ and $\mathrm{iOAB} \cdot{ }^{8-10}$

The urethra is predominantly innervated by the pudendal nerve that originates from S2-S4 in the spinal cord (Figure 2). From there, the branches go through the Alcock's canal into the areas deep in the pelvis. This somatic nerve uses ACh and nicotine receptors for signaling. The nerve is mostly known for its motor control of the EUS, but it also innervates the external rectal sphincter, the scrotal muscles and the perineal area. There is also a branch called the dorsal genital nerve (DGN) that innervates the penis or clitoris and is important for sexual function (Figure 1). Besides efferent nerves, the pudendal nerve has many afferent fibers that sense urine flow through the urethra. ${ }^{1}$

The EUS is essential for normal urinary tract function and it is under voluntary control, which means that a person can decide to contract and relax the striated muscle fibers of their sphincter and to interrupt voiding. These motor nerves originate in a specific area in the ventral horns of the sacral spinal cord called Onuf's nucleus. ${ }^{1,3}$

Inhibition of voiding is done with a third peripheral nerve called the hypogastric nerve that originates from the T11-L2 region in the spinal cord (Figure 2). The branches of this sympathic nerve innervate the bladder dome and
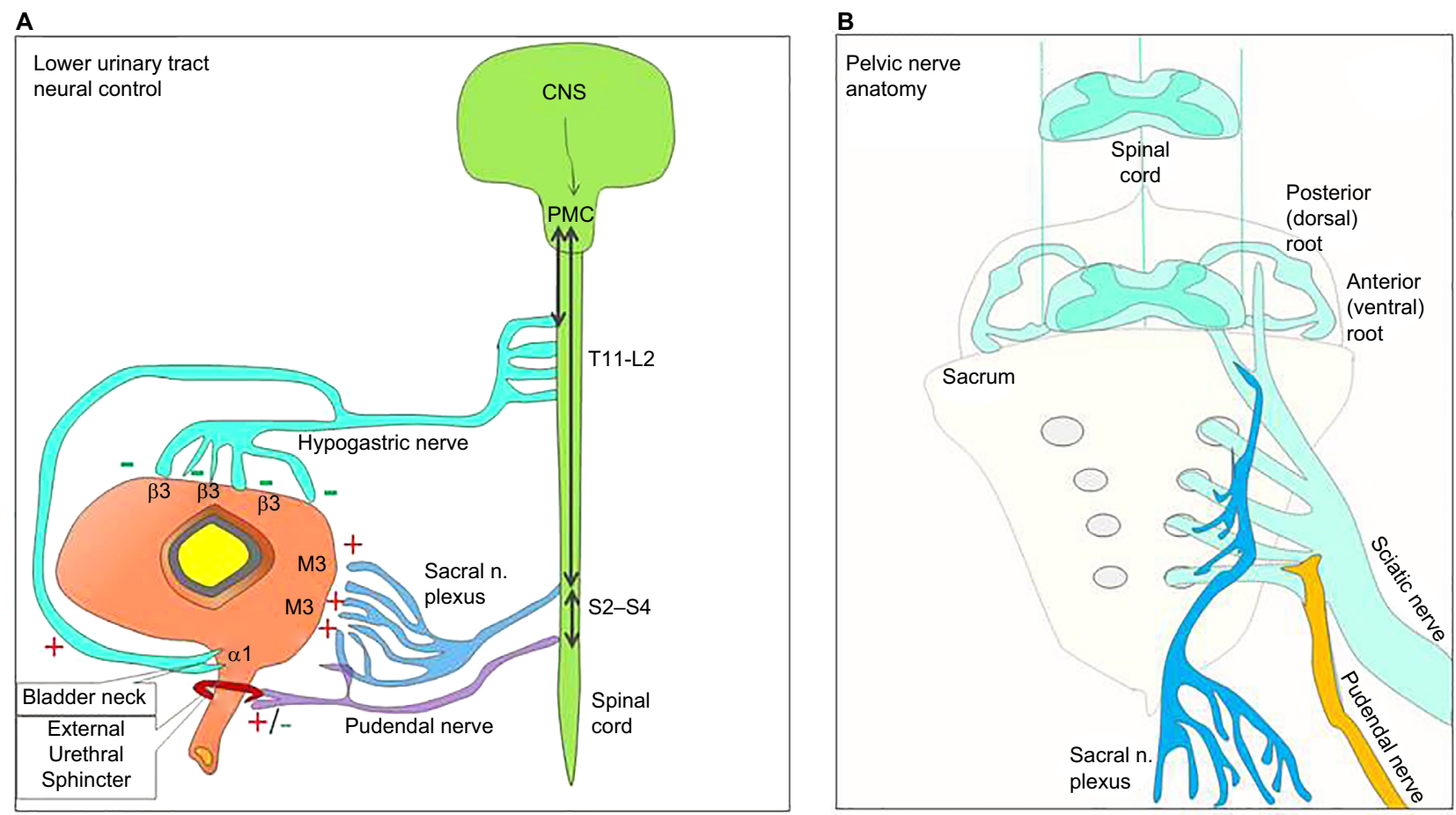

Figure 2 Pelvic nerve anatomy and lower urinary tract neural control.

Notes: (A) A model of the different nerves that innervate and control the lower urinary tract. (B) Pelvic nerve anatomy.

Abbreviations: CNS, central nervous system; PMC, pontine micturition center. 
bladder neck and use predominantly norepinephrine (noradrenalin) as a neurotransmitter. In the bladder dome, it activates $\beta 3$-receptors that inhibit detrusor contractions (thereby inhibiting cholinergic activation). In the bladder neck, it does the opposite and activates $\alpha 1$-receptors that contract the striated muscles of the internal sphincter. Both $\beta 3$ - and $\alpha 1$-receptors are used for pharmacological therapy (Figure 2). $\beta 3$-agonists relax the detrusor, and $\alpha 1$-receptor antagonists are used to relax the proximal urethra (bladder neck) in outflow obstruction patients.

The central neural control for storage and voiding is located in the brain stem, where lies the periaqueductal gray (PAG) and pontine micturition center (PMC). The PMC is inactive in the storage phase during which the bladder sacral efferents are inhibited, resulting a relaxed detrusor, combined with a contraction of the bladder neck and EUS. ${ }^{11}$ The PAG receives afferent information from the lower urinary tract and further communicates with the higher brain centers including the forebrain. The PAG is the site that the central nervous system targets to initiate voiding (Figure 2). When afferent signals in the PAG reach a certain threshold and there is permission to void (implying no inhibition from higher brain centers), it activates the PMC to initiate voiding reflexes.

During storage and voiding, bladder and sphincter coordination is based on reflex mechanisms. ${ }^{1,3}$ Two normal reflexes are active in the storage phase. The storage reflex includes the hypogastric nerve to initiate smooth muscle fiber contraction in the bladder neck and inhibits detrusor smooth muscle activation by the sacral efferent nerves. The second reflex is the guarding reflex. This sacral reflex increases sphincter tonus during sudden abdominal pressure increases to maintain continence. ${ }^{12}$ The spinobulbospinal or somatic micturition reflex is a voiding reflex. This reflex is activated at start of a void and is maintained throughout voiding and works by 1) inhibiting the guarding reflex, 2) coordinating the voiding contraction of the detrusor and 3) relaxing the bladder outlet. ${ }^{11}$ The spinobulbospinal reflex is triggered by afferent firing due to flow in the urethra and the voiding contraction of the bladder. The PMC plays the central role in initiating and maintaining this reflex. Besides these normal reflexes, neurologic disease or inflammation can cause pathologic or noxious reflexes that decrease the inhibitory control of the bladder.

The synchronization of the nerves that controls the lower urinary tract is mediated through different peripherally and centrally located ganglion cells. ${ }^{1}$ The sacral nerves innervate the bladder wall through the pelvis and form a large part of the pelvic plexus. The pelvic plexus contains different auto- nomic ganglia that signal between ganglia in 1) the bladder wall (intramural), 2) the pelvis, 3 ) the posterior (dorsal) and anterior roots, 4) the spinal cord and 5) the brain. Connecting the afferent and the efferent systems is done with interneurons in the spinal cord. These interneurons relay afferent and efferent signals to and from the brain, but there are also interneurons that have inhibitory or excitatory synapses between afferent and efferent neurons within the spinal cord. ${ }^{1}$ Besides the sacral nerves, the pelvis also contains other nerves that innervate the EUS, rectum, uterus, genitals and nerves that descend toward the lower extremities. There are three proposed pathways for cross talk between visceral pelvic organs and cross-sensitization. ${ }^{13}$ The first pathway is the interaction at the spinal cord level. The nerves in the pelvis use many communal sensory and motor roots of which many lie in the sacral spinal cord. ${ }^{14}$ For instance, afferent fibers form sacral and pudendal nerves that enter the dorsal horns of the sacral spinal cord at a similar level. ${ }^{1}$ The second proposed pathway can be described as descending modulation by higher brain centers. ${ }^{15,16}$ This pathway describes how higher brain centers can modulate spinal nociceptive processing, which occurs after exposure to visceral pain for long periods of time or during period of chronic stress. ${ }^{15}$ The third pathway is cross talk through peripheral sensitization. There are multiple animal studies that show that afferent pain signals from one organ can induce a neuroinflammatory response in another pelvic organ. ${ }^{17,18}$ Studies by Pezzone et $\mathrm{al}^{19}$ and Winnard et $\mathrm{al}^{17}$ show that this could be possible because some DRG neurons have multiple axons or dichotomizing axons that innervate different pelvic organs. This cross talk between pelvic organs gives an explanation for the correlation between bladder pain and other somatic disorders that are part of chronic pelvic pain such as irritable bowel syndrome.

\section{Neuromodulation}

Continuous or intermittent electrical nerve stimulation strategies are used to treat lower urinary tract dysfunction. Neuromodulation is used to treat both DO and detrusor underactivity, but it is also currently used to treat bladder pain. Electrical stimulation is performed at different sites of the human body and therefore targets different nerves (Figure 1). Some of these target nerves are directly involved in lower urinary tract sensory-motor control, such as the sacral or pudendal nerves, while others are more indirectly involved, such as the DGN and the posterior tibial nerve (PTN). The PTN is a distal branch of the sciatic nerve that originates in the pelvis (L5-S3 spinal roots) and descends toward the lower extremities (Figures 1 and 2). Stimulating 
the PTN has an effect on lower urinary tract function. Chinese acupuncturists have been applying stimulation in the area of the PTN to treat lower urinary tract dysfunction for centuries. Nowadays, electrical stimulation of the PTN is an established therapy to treat lower urinary tract dysfunction with proven clinical efficacy. ${ }^{20,21}$

Much of what is known about the biologic effects of electrical stimulation therapies for lower urinary tract dysfunction comes from animal studies that investigated how neural stimulation interacts on the different peripheral, spinal and higher CNS pathways. Most of these studies show that electrical stimulation of the sacral nerve roots or the peripheral nerves will not inhibit the primary efferent nerves directly but operate by modulating afferent nerve signaling. ${ }^{22-24}$ Zhang et al demonstrated in a feline model that it was the direct stimulation of the S1-S3 dorsal roots and not the ventral roots that inhibit bladder contractions. ${ }^{22}$ A similar effect is seen when SCI patients with severe DO undergo a dissection of the dorsal (sensory) roots (rhizotomy) to successfully inhibit DO. ${ }^{25}$ This suggests that electrical stimulation has an indirect effect that modulates interneural transmission pathways. ${ }^{22}$ The effect of electrical stimulation in different locations such as the sacral, pudendal or tibial area could therefore result in modulation of different pathways or have a different effect on a single pathway. The study from Xiao et $\mathrm{al}^{24}$ provides interesting clues for this. They performed pudendal nerve stimulation and tibial nerve stimulation in felines with transected sacral spinal cords and demonstrated that pudendal nerve stimulation was still successful in inhibiting bladder contraction amplitudes, but tibial nerve stimulation was not. This implies that for lower urinary tract function, pudendal nerve stimulation acts on lower sacral reflex pathways, and tibial nerve stimulation modulates another pathway that includes suprasacral spinal cord regions such as the brain.

Other evidence for central nervous effects of neuromodulation in humans comes from functional magnetic resonance imaging studies. ${ }^{26,27}$ Kavia et $\mathrm{al}^{27}$ demonstrated in Fowler syndrome patients that these subjects had an overall low activity in the brain areas that process bladder afferent signals such as the PAG, but these regions could however be reactivated with sacral neuromodulation. Blok et $\mathrm{al}^{26}$ showed that continuous electrical nerve stimulation in the sacral area with sacral nerve stimulation (SNS) modulates the brain regions that are associated with learning behavior. These include areas that are associated with detrusor hyperactivity and areas for alertness and awareness. This implies that neuromodulation gives patients more control over their bladder. The clinically applied neurostimulation techniques and the current evidence for these therapies will be explained in the next section.

\section{Sacral anterior root stimulation (SARS)}

SARS was already developed in the sixties by Brindley and clinically applied in SCI patients in the early eighties. ${ }^{25,28,29}$ Electrical currents are used to activate sacral anterior (motor) roots of S2, S3 and S4 spinal cord segments to accomplish, respectively, voiding, defecation and erections in complete SCI patients who have lost all these functions. The device itself is nowadays called the Finetech-Brindley System (Finetech Medical Ltd., Welwyn Garden City, UK). It requires 1) surgically implanted electrodes that are attached to the anterior roots, 2) an internal receiver and 3) an external controller with a transmitter block (Figure 3). The initial surgery for the placement of the electrodes can be performed intradural and extradural in both open and laparoscopic procedures. An essential step of the surgical procedure is the isolation of the dorsal (sensory) and anterior (motor) roots of spinal cord segments S2, S3 and S4. The intradural procedure requires a sacral laminectomy to accomplish this. After this, the spinal cord roots are stimulated individually with electrical currents to investigate their function. Dorsal sensory roots form a reflex arch with the anterior motor roots, and this influences motor control over the bladder. Leaving the dorsal roots intact therefore leads to persistent DO. Therefore, a rhizotomy is done to surgically dissect the dorsal (sensory) roots. Despite the benefits of preventing bladder reflex activity and hardly any side effects in patients with complete spinal cord lesions, the patients are sometimes reluctant to undergo this necessary surgical procedure. Martens et a $\mathrm{l}^{30}$ evaluated reasons for this, and patients reported the irreversible damage to the dorsal roots caused by the procedure as one of the main motives for not undergoing the procedure. After the rhizotomy, the designated anterior roots are organized and fixated in an electrode book that contains the electrodes to stimulate the different motor roots separately (Figure 3 ). From there, the electrical wires are tunneled to a subdermal receiver on the abdominal side. Patients have to be able to localize this area easily. The internal receiver has a pulse generator and is activated by a radiofrequency transmitted signal from an external transmitter block. This is done by placing the external transmitter block on the skin overlapping the site of the internal receiver. The remote control to which the external transmitter block is connected has settings for different programs that can be altered for voiding, defecation and erection function. Individual fine tuning of the programs 

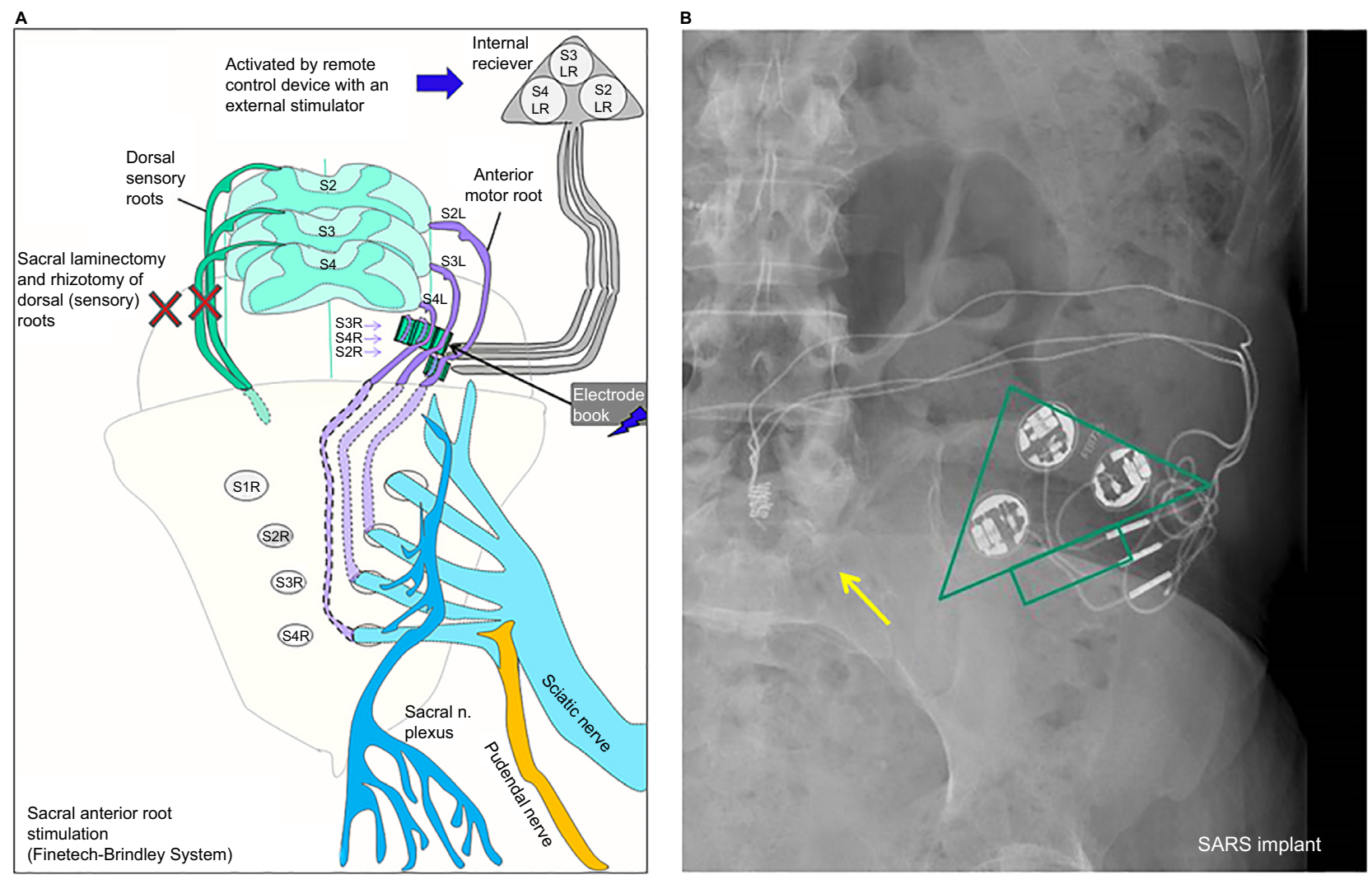

Figure 3 SARS therapy for spinal cord injury patients.

Notes: (A) How anterior roots S2, S3 and S4 are connected to electrodes in an electrode book. The electrodes are guided to an internal receiver (called a Chimney procedure). The receiver can be activated with an external controller. The two "X" depict a laminectomy to gain access to the nerves (for the intradural procedure) and a rhizotomy of the dorsal horn of S2-S4. (B) An X-ray image (AP) of the upper pelvic area of a patient with a Finetech-Brindley System. The yellow arrow depicts the electrode book where the electrodes are connected to the anterior roots of S2, S3 and S4. The wire electrodes are guided to the internal electrical pulse receiver plate that is shown as the green triangle.

Abbreviations: AP, anterior posterior view; SARS, sacral anterior root stimulation.

is possible with alterations in the pulse frequency, intensity and duration for each of the three anterior roots. Voiding is however different compared to the normal situation. Electrical stimulation of sacral anterior roots contracts the smooth muscle fibers of the detrusor and also the striated muscles of the bladder neck and urethral external sphincter. Continuous stimulation would therefore result in a dyssynergia between bladder and sphincter coordination during voiding. This problem is overcome by burst stimulation. After each burst, there is a fast relaxation of the striated sphincter muscles, but a much slower relaxation of the detrusor smooth muscles, resulting in bladder emptying between the stimulation bursts. This gives an intermittent voiding pattern that is called poststimulus voiding.

The Finetech-Brindley System can significantly improve the quality of life and independence of spinal cord patients. ${ }^{29,30}$ Getting control over the lower urinary tract with this device can reduce or abolish the need for bladder catherization and reduce the number of urine tract infections. ${ }^{29,30}$ But the device can also decrease constipation problems and restore some of the erectile function in patients. ${ }^{29,30}$ Incontinence, for example, is improved with $50 \%$ of patients who are fully continent with the device compared to $13 \%$ of a control group. ${ }^{30}$ Martens also evaluated quality-of-life aspects in this study with a cohort of 46 patients with a Finetech-Brindley System and $28 \mathrm{SCI}$ controls. The study showed that the patients with SARS have a significantly better feeling of general health and social functioning and also less limitations, anxiety and bad feeling concerning their urinary problems. ${ }^{30}$

It is, however, important to address and manage patient's expectations. The initial surgery is a lengthy procedure and can cause anterior root damage and cerebrospinal fluid leakage. ${ }^{25,28}$ Surgery is also not always successful for all three functions. For instance, Brindley and Rushton ${ }^{29}$ reported that after 5-11 years, only one-third of male patients get implant driven erections. The Martens et $\mathrm{al}^{30}$ study showed poorer outcomes and reported successful erectile function in only $0 \%-32 \%$ of patients. Apart from this, the patients have to be consulted that the therapy does not bring back genital sensations. ${ }^{30}$ 
The average life span of the technical equipment is reasonably good. The device is quite robust and does not use any internal battery. Brindley and Rushton ${ }^{29}$ showed in a long-term follow up study (5-11 years) in 50 patients that 41 patients were still using their device for voiding and 27 for defecation. Martens et a ${ }^{30}$ however showed lower scores in a group of over 70 patients ( $63 \%$ still using the device). Technical failure therefore does happen and restoration requires renewed surgery in a group of patients who have an increased health risk. ${ }^{29,30} \mathrm{~A}$ factor that also has to be taken into account is that program settings sometimes need to be adjusted over time. This can be caused by either technical problems with the system or possibly the slow changes in lower urinary tract function in SCI patients. Overall, patient satisfaction with SARS is high and is currently the only option for complete SCI patients to regain neurologic control over their lower urinary tract. ${ }^{30}$

\section{SNS}

SNS is a neurostimulation technique that has evidence-based clinical applicability. SNS aims to restore bladder neural control for both $\mathrm{OAB}$ and urinary retention. ${ }^{8,31,32}$ The technique was developed by Tanagho and Schmidt in the late eighties for neurogenic bladder disease. In Europe, the device was implanted for the first time in 1989 by van Kerrebroeck (Figure 4). Later on, this technique was investigated for the treatment of fecal incontinence for which it was also FDA approved in the nineties. The SNS technique involves the placement of an implantable electrode through the sacral foramen of typically S3 to stimulate the sacral and pudendal nerves (Figure 4). The electrode is tunneled subcutaneously and connected to a surgically placed internal pulse generator (Figure 4). The InterStim ${ }^{\circledR}$ device (Medtronic, Minneapolis, MN, USA) is currently the only device that is clinically used, and according to the manufacturer, over 1,750,000 devices have currently been implanted.

When SNS was developed, the initial thought was that targeting the pudendal nerve with electrical stimulation would help contract the EUS and pelvic floor. However, urodynamic studies showed that SNS was actually very potent in inhibiting DO. Like stated before, the main hypothesis now is that SNS inhibits DO not by direct inhibition of the efferent motor nerves to the detrusor but by modulating the lower urinary tract reflexes via the afferent nerves and interneurons in the spinal cord. ${ }^{14,33,34}$ Electrical stimulation of the afferents from the bladder and sphincter is thought to correct the unbalanced neural signaling that causes the lower urinary dysfunction. This latter hypothesis also explains why
SNS can inhibit DO and activate the detrusor in patients with urinary retention.

Before patients undergo a surgical implantation of the InterStim device, a testing phase is performed to evaluate whether the therapy is successful. This test phase is called the percutaneous nerve evaluation (PNE). During the PNE procedure, a hollow needle electrode is transcutaneous inserted through the different sacral foramina of S2, S3 and S4 in a conscious patient. Positioning and stimulation of the needle is done to evaluate 1) the sensations of the patient that are ideally described as nondisturbing tingly sensations in genital or perineal areas and 2) motor reflexes such as the bellows reflex (levator ani contraction visible in perineal area) and flexing of the big toe (PTN reflex). After choosing the optimal position, the needle will be replaced by 1) a tined electrode (e.g., tined lead) with four stimulation points (Figure 4) or 2) a wire electrode with one stimulation point (Figure 1). These electrodes will be connected to an external stimulator device that generates continuous electrical pulses that can be adjusted in intensity and frequency. Testing can be done with one electrode or with two bilaterally placed electrodes. ${ }^{35}$ The Pham study reported significantly higher success rates of the PNE test in patients who were evaluated with bilateral electrodes, but these results were not supported by an earlier study by Scheepens et al. ${ }^{36,37}$ After the procedure, the patients are evaluated to objectify how much improvement is in their symptoms with SNS. This is done with voiding diaries and urodynamic investigations (especially in patients with urinary retention). Successful treatment of the therapy is defined as a $50 \%$ improvement in symptoms. Kessler et al $^{38}$ reported that the use of the tined electrode was less prone to migration and allowed for longer testing periods resulting in higher success rates of the PNE test. The investigators advised a testing period of 14 days. If the PNE is evaluated as effective and a wire electrode was used for the procedure, it will be replaced with a tined electrode (e.g., tined lead). The wire of the electrode is tunneled under the skin and connected to a surgically implanted internal pulse generator that contains the battery and works similar to a pacemaker. ${ }^{39}$ This latter device can be magnetically activated and accessed via a remote control for periodical read outs of the machine and to change settings when needed.

SNS has proven efficacy in iOAB, neurogenic bladder disease, hypocontractility of the bladder and fecal incontinence. ${ }^{40-42}$ It is currently being evaluated in patients with chronic pelvic pain syndrome and chronic constipation. The advantages of this device are that it applies continuous stimulation to improve lower urinary tract control and that 

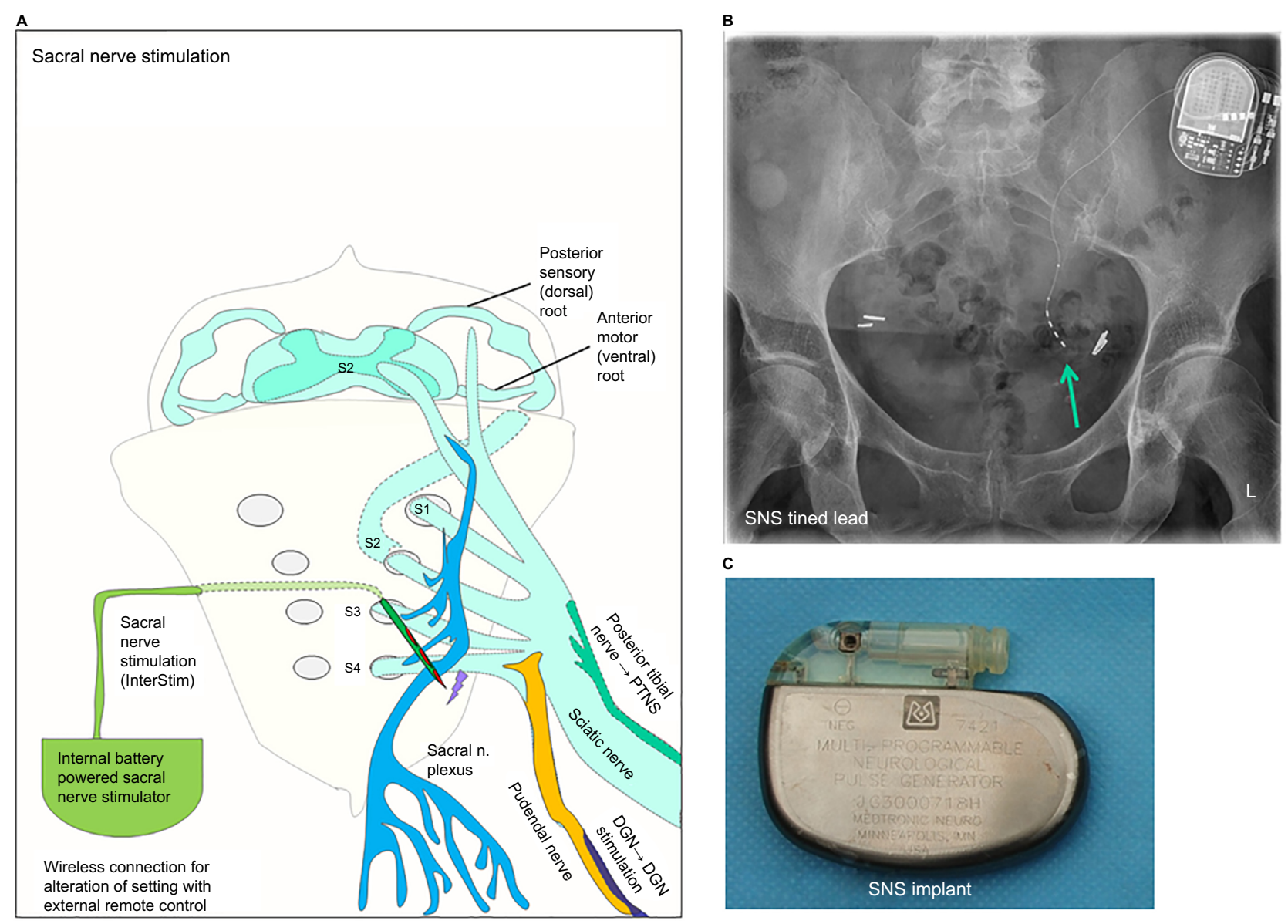

Figure 4 Sacral nerve stimulation with InterStim ${ }^{\circledR}$ device and pelvic anatomy for dorsal genital nerve (DGN) stimulation and percuteneous tibial nerve stimulation. Notes: (A) A schematic diagram of how sacral nerve stimulation (SNS) works with a tined lead electrode that is placed through sacral foramen S3. The nerve fibers from the posterior tibial nerve are part of the large sciatic nerve that descends from the pelvic region toward the lower extremities. Also the pudendal nerve is shown, which contains the nerve fibers from the DGN, which branches from this nerve and can be used for electrical DGN stimulation. (B) An X-ray of the pelvic region from a patient with an InterStim SNS device with the green arrow pointing at the tined lead. The pulse generator is seen in the upper right corner of the image. (C) An image of an SNS device that was explanted. This was the first SNS device that was implanted in Europe in 1989.

Abbreviations: DGN, dorsal genital nerve; SNS, sacral nerve stimulation.

the device is internally located. Battery life is on average 5-7 years but depends on 1) the system (there is a large battery and a small battery system) and 2) how energy efficient the internal device settings are (pulse intensity and frequency $^{31}$ ). The internal InterStim stimulator (the pacemaker) does have to be surgically replaced when the pacemaker battery is empty, but the tined electrode can be left in place. van Voskuilen et a ${ }^{31}$ investigated long-term results of SNS in 149 patients in a single-center study with a mean follow-up of 63 months. This study reported a considerable rate of 129 reoperations and 21 explanted of the stimulators. Infection problems occurred in only six patients, resulting in surgical removal of one device. van Kerrebroeck et al undertook a large multicenter study in 152 patients with refractory $O A B$ $(n=121)$ or urinary retention $(n=31)$. They reported 36 devicerelated technical failures requiring surgery in 60 patients. These 60 patients underwent an additional 110 surgeries during a 5-year time span. ${ }^{40}$ Nonetheless, $70 \%$ of the patients experienced therapeutic benefit from the treatment after 5 years, and this was in a group that was refractory to other therapies. Treatment failure occurred mostly within the first 6-24 months after placement. For refractory OAB, long-term efficacy was shown with incontinence episodes reducing $58 \%$ and the number of voids per day $40 \% .{ }^{40}$ Siegel et a ${ }^{43}$ performed a prospective multicenter in $272 \mathrm{OAB}$ patients (90\% female) who received SNS therapy for an average of 3 years. This study showed similar or even better success rates compared to the van Voskuilen and van Kerrebroeck studies. This study also reported a very consistent improvement in quality of life with $52 \%$ of patient reporting that interference of urinary symptoms on everyday life was greatly reduced and a significant improvement was seen on coping, social, sleeping and health-related quality-of-life subscales. ${ }^{43}$ van Kerrebroeck et a ${ }^{40}$ also evaluated SNS efficacy in 31 patients 
with urinary retention. SNS therapy was considered successful in $71 \%$ of the patients, and voided volumes increased considerably in this group, which was shown by an average reduction of catherizations/day from 5.3 to 1.9 and a decrease of catherized volumes from $380 \mathrm{~mL}$ to $109 \mathrm{~mL}$.

\section{Percutaneous posterior tibial nerve stimulation (P-PTNS)}

P-PTNS is a low invasive neuromodulation technique that intermittently stimulates the easily accessible PTN at the ankle with a transcutaneously placed needle electrode ${ }^{44,45}$ (Figure 1). Electrical stimulation of the PTN for bladder dysfunction was first attempted by McGuire et $\mathrm{al}^{46}$ and the percutaneous technique was developed by Stoller. P-PTNS resembles traditional acupuncture techniques but combines it with electrical pulse stimulation. Like stated before, P-PTNS indirectly intervenes in the neural pathways of the lower urinary tract and most likely modulates higher spinal and/or brain reflexes to treat OAB symptoms. ${ }^{24,47,48} \mathrm{P}-\mathrm{PTNS}$ is also experimentally investigated for other indications such as urinary retention, pelvic pain, pediatric bladder dysfunction, fecal incontinence and anal fissures. ${ }^{49}$ The therapy comprises weekly clinical visits for 30-60 min stimulation sessions for up to 12 weeks. Pulse intensity and frequency can be adjusted to achieve optimal response in each individual patient. After this initial 12-week period, monthly visits are recommended to maintain the desired treatment effect.

The full mechanism of action of P-PTNS is still not fully understood, and the therapy was initially received with skepticism by physicians. This was until Finazzy-Agr et $\mathrm{al}^{50}$ and Peters et $\mathrm{al}^{21,51,52}$ demonstrated true efficacy in two randomized, placebo-controlled trials. The therapy is currently included in the guidelines for OAB treatments from the International Continence Society, American Urological Association, and European Association of Urology. ${ }^{10}$

The SUmiT trial investigated P-PTNS treatment in 220 patients with OAB in a double-blind, sham-controlled randomized trial. ${ }^{21}$ Of the treatment group, 54\% reported moderate to severe improvement in symptoms, compared to $20 \%$ in the sham group. ${ }^{21}$ Although there was a large placebo effect, the treatment arm reported a significant reduction in moderate to severe urgency episodes from 8.3 to 3.7 , which was significantly better compared to the sham-treated group (8.0-5.0). Similar improvements were reported in incontinence episodes per day with a significant reduction in the treatment arm (3.0-0.3) compared to the sham-treated group (1.8-1.0). For initial responders, the overall long-term efficacy was good with a reported $96 \%$ sustained effect after
1 year. ${ }^{53}$ Regarding safety, Peters et $\mathrm{al}^{21}$ reported that the needle insertion and electrical stimulation could create mild local discomfort, but adverse events from P-PTNS therapy such as local bruising, bleeding and discomfort all occurred in $<1 \%$ of patients.

\section{Wireless internal tibial nerve stimulation}

This technique is based on the same principles as P-PTNS in combination with the wireless electrical pulse generation technique that is used in the SARS Finetech-Brindley System. The stimulation of the PTN is achieved with a small, surgically implantable electrical pulse transmitter with electrodes that are attached near the PTN at the ankle (Figure 1). There is a battery-free transmitter that converts the power and control signals from an extracorporeal controller into electrical pulses that are guided through the stimulation electrodes. This extracorporeal controller is placed on the skin near the receiver, and radio frequency transmission is used to transfer energy to the receiver. The controller settings can be adjusted for altering pulse frequency and intensity, and the system can be independently operated by patients for on demand PTN stimulation. The Urgent-SQ ${ }^{\mathrm{TM}}$ (Cogentix Medical, former company Uroplasty, Minnetonka, MN, USA) was the first implant that was developed, and it was evaluated in eight patients with refractory $\mathrm{OAB}$ and a positive response on P-PTNS treatment in $2003 .{ }^{54}$ This device was developed for ambulant use to reduce the burden and costs of the mandatory clinical visits that are part of the conventional P-PTNS therapy. Furthermore, the added freedom of on-demand stimulation could improve the overall efficacy of PTN stimulation. Initial results of this pilot were promising, with a $50 \%$ improvement in symptoms in half of the patients after 1 year. In one patient, the implant was removed within the first year due to technical failure and one patient had discomfort at the site of the implant. A 9-year follow-up study was conducted in this patient cohort. ${ }^{55}$ The results showed that one patient lost the usual sensory and motor responses during stimulation after 4 years. It was concluded that this was a probable technical failure. Of the six remaining patients, three were still using the device on a regular basis with consistently improvement in quality of life compared to baseline. These three patients would also recommend the therapy to others. The device was never clinically introduced on a large scale. However, since the arrival of more scientific evidence that supports P-PTNS therapy, interest for these ankle implants has gained. A clinical trial was recently initiated to evaluate a new device called the BlueWind Medical Renova ${ }^{\mathrm{TM}}$ (BlueWind Medical Ltd, Herzliya, Israel) (Figure 1). This device uses the same principles 
and technology, but it is much smaller than the Urgent-SQ ${ }^{\mathrm{TM}}$ and has no wire electrodes (Figure 1).

\section{Percutaneous DGN stimulation}

DGN stimulation with needle electrodes is an experimental neuromodulation technique to treat neurogenic and $\mathrm{OAB}$ disease, and it is not applied in routine clinical practice. ${ }^{56}$ Like mentioned before, the DGN is a branch of the pudendal nerve $^{57}$ (Figures 1 and 4). Stimulating the DGN may have several advantages compared to other frequently stimulated nerves such as the sacral nerves and the PTN. For instance, the DGN is, such as the PTN, easily accessible with a small percutaneously inserted needle electrode but can also be accessed with a wire electrode that is guided through a hollow needle. ${ }^{56}$ The nerve is also a direct branch of the pudendal nerve and has therefore, in theory, a shorter access to the nerves that control lower urinary tract function compared to the PTN. Successful stimulation of the DGN can be detected by monitoring the presence of the genitoanal reflex. ${ }^{56}$ Martens et $\mathrm{a}^{56}$ experimented with percutaneous DGN stimulation in six SCI patients with DO and demonstrated with urodynamics that DO can be inhibited with this technique. Goldman et a ${ }^{58}$ investigated DGN stimulation in 21 female OAB-wet patients with a percutaneous wire electrode. Patients received 7 days of continuous stimulation, and all but two completed the study ( $\mathrm{n}=19)$. In $47 \%$ of the patients, there was a reduction in incontinence episodes/day of $>50 \%$, and $81 \%$ of patients had a reduction of severe urgency episodes/day of over $50 \%$. The results of a pilot study by van Breda et al also show promise. ${ }^{59}$ This study investigated on demand DGN stimulation through a percutaneously placed wire electrode in six patients with non-neurogenic OAB. Patient reported $73 \%$ improvement in symptoms, including urgency an incontinence severity. Larger study cohorts are needed to determine the applicability of percutaneous DGN stimulation. Transcutaneous electrical nerve stimulation (TENS) of the DGN has also been investigated (refer the "TENS" section).

\section{Pudendal nerve stimulation}

To target the pudendal nerve more specifically, Spinelli et $\mathrm{a}^{60}$ described a surgical procedure that places an electronic lead into Alcock's canal via either a perineal approach or a posterior approach. The technique uses the same InterStim device with a tined lead for continuous nerve stimulation as in SNS. The hypothesis was that targeting the pudendal nerve alone would potentially help neurogenic bladder patients in which SNS did not work sufficiently. ${ }^{61}$ Spinelli et al performed a pilot study in 15 patients with neurogenic bladder disease and reported encouraging results with a decrease in incontinence episodes/day from 7 to 2.6. This coincided with a twofold increase in bladder capacity and a positive effect on constipation meaning that stool evacuation improved from 2.6 to 7 times/wk.

Groen et $\mathrm{al}^{62}$ investigated the applicability of a mini neurostimulator called the Bion ${ }^{\circledR}$ that was placed near the pubendal nerve in Alcock's canal. This pilot study of six patients was successful in significantly decreasing the degree of incontinence, with no severe adverse event. Surgical access and vascular control in women but especially in men was considered challenging. Despite the positive clinical outcomes, no follow-up study of this device has been done. Peters et $\mathrm{al}^{63}$ investigated whether isolated pudendal nerve stimulation was superior to SNS with a cross-over study in 30 patients. Results were remarkably in favor for pudendal nerve stimulation and were chosen as the superior lead in $79.2 \%$ with an average reduction of symptoms of $63 \%$ compared to $46 \%$ for the SNS group. A total of $80 \%$ of the patients who were evaluated had a positive response and received a permanent implant for pudendal nerve stimulation with only minimal complications reported. Wang et al performed intermittent electrical pudendal nerve stimulation with long percutaneously placed needle electrodes. This group performed a longterm efficacy study of at least 60 months in 106 patients with urgency-frequency who received on average 21.6 stimulation sessions.$^{64}$ The study reported an $85 \%$ of patients who had an improvement in symptoms of over $50 \%$. In general, pudendal nerve stimulation is still not a routinely applied therapy. Especially the study results by Spinelli et $\mathrm{l}^{60}$ and Peters et $\mathrm{al}^{63}$ in 2005 seem promising, but more evidence from larger study cohorts with long-term follow-up is needed to prove clinical superiority of pudendal nerve stimulation over SNS.

\section{TENS}

The TENS technique was developed in the 1970s to relieve pain. Dermal patch electrodes are used to transcutaneously stimulate internal nerves. Sudin introduced this technique in urological patients in 1974, and it is currently applied for patients with $\mathrm{iOAB}$, neurogenic $\mathrm{OAB}$ and pelvic pain syndrome. The technique can be applied in different regions to target different nerves that are involved in lower urinary tract control and can be used for continuous or on-demand stimulation.

The anatomical areas where TENS is applied include 1) the sacral area to stimulate sacral and pudendal nerves, 2) the ankle to stimulate the PTN and 3) the genital/groin area to stimulate the DGN and/or pudendal nerve. The real 
advantages of this technique are 1) the use of dermal patch electrodes that are noninvasive and 2) the possibility for patients to learn and apply the technique themselves. Fjorback et $\mathrm{al}^{65}$ reported that on-demand stimulation of sacral nerves with TENS in neurogenic bladder patients with multiple sclerosis achieved some inhibition of DO, but authors concluded this worked insufficiently for clinical use. A large systematic review was performed to evaluate the efficacy of TENS for neurogenic bladder disease. ${ }^{66}$ This study included 22 studies with a total of 450 patients. Conclusions of this systematic review were for a large degree based on the results from a randomized controlled trial by Guo et al who investigated sacral stimulation in 61 poststroke patients with incontinence. This study showed a significant improvement in incontinence episodes per day from 4.0 to 1.6 in the treatment group compared to 4.2 to 3.86 in the control group. ${ }^{67}$ The authors of the systematic review reported that many of the included studies were of poor quality, but they did conclude that TENS appeared to be effective at reducing symptoms and that the technique appeared safe. ${ }^{66}$

DGN stimulation with TENS was investigated in a small group $(n=7)$ of patients with neurogenic DO by van Breda et al. ${ }^{59}$ The study reported that DGN stimulation with TENS increased bladder capacity and voided volumes. ${ }^{59} \mathrm{~A}$ urodynamic study also reported that DGN stimulation with dermal patch electrodes can inhibit DO in neurogenic patients. ${ }^{68}$ Nonetheless, larger studies are needed to demonstrate whether DGN stimulation with TENS has an real effect on OAB. ${ }^{69}$

The overall clinical evidence for the use of PTNS and SNS for treating $\mathrm{OAB}$ is more convincing compared to TENS. The skin is a large barrier to overcome for electrical stimulation, which is a clear downside of the TENS technique. Nonetheless, the use of dermal patch electrodes does give practical advantages over PTNS and SNS. Because TENS is noninvasive, it is potentially more suitable for pediatric patients, although this is still considered experimental. Because of the advantages that TENS has over other therapies, it has obtained a niche in clinical practice for bladder dysfunction treatment.

\section{Magnetic nerve stimulation}

Magnetic nerve stimulation is an extracorporeal noninvasive technique that can transmit electrical currents to activate nerves in deeper tissue layers. It is a painless technique, but this type of stimulation can cause discomfort. The technique itself was developed in Sheffield and uses magnetic fields to generate electrical currents in the body. ${ }^{70}$ In daily practice, it is sporadically used for pelvic floor muscle training in women with stress urinary incontinence (SUI). Fujishiro et $\mathrm{al}^{71}$ performed a sham-controlled trial evaluating the efficacy of magnetic nerve stimulation of the pelvic area in 62 patients with SUI. Only a single 30-min stimulation session with a magnetic coil was performed in this study. The follow-up was for 1 week. The study reported a reduction in total amount of incontinence episodes in 3 days from 4.3 to 2.2 in the treatment group $(n=31)$, which was significantly more than the sham-treated group ( 3.9 to $3.2 ; n=31$ ). Recently, some trials have been conducted in patients with OAB. Lo et $\mathrm{al}^{72}$ investigated magnetic nerve stimulation in 49 patients with SUI and 44 patients with OAB in a retrospective study. A electromagnetic chair was used for stimulation two times per week for 9 consecutive weeks. In all, 21 patients discontinued treatment. The results from the patients who completed the treatment showed that $48 \%$ of the $\mathrm{OAB}$ patients were symptom free (no urgency, frequency or incontinence) after treatment and $33 \%$ of SUI patients were symptom free (no incontinence). Although these are promising results, we do not know how long the effects last, and the results should be investigated in a larger study cohort. The technique is also not very specific in targeting individual nerves. Magnetic nerve stimulation is currently not widely used in clinical practice, and further research is mandatory to determine true clinical (long term) efficacy.

\section{Summary}

For decades, neurostimulation and neuromodulation therapies have been a validated and widely used treatment option for lower urinary tract dysfunction. The neurostimulation techniques that are currently available vary from very low invasive treatments to more high invasive therapies that require elaborate surgery. Some experimental techniques look promising but lack follow-up studies in larger cohorts to determine efficacy. This is important because placebo effects are often considerable in studies that investigate bladder dysfunction treatments.

Devices are improving from a technical point of view and are becoming more refined. But there is still room for improvement. Internal stimulator devices are foreign bodies and are therefore at risk of infection. In a wet and dynamic environment, technical failure can occur. The future directive for this field is to fine tune these devices. For stimulators, there is improvement in battery life and finding ways for external recharging of batteries to avoid resurgery. Improving the size and robustness of the apparatus and electrodes and making the surgical placement easier are factors that could improve efficacy, durability and tolerability of these therapies. For the intermittent neuromodulation therapies 
such as PTNS, techniques should be focused on improving the independence of the patient with more home-based and ambulant therapies. The potential wireless internal tibial nerve stimulation technique is a good example for this.

Neuromodulation has a proven efficacy in refractive OAB patients who do not respond to pharmacological therapies. It has improved the quality of life of many patients who suffer from lower urinary tract dysfunction and urinary incontinence. Neurostimulation therapies such as SARS helped to dramatically increase independence for wheelchair bound spinal cord patients. Indications for different types of neuromodulation therapies are broadening since new studies reveal efficacy of these therapies in other conditions such as pelvic pain. Neurostimulation in urology has been a pioneering field and is an example of how novel engineering techniques can be picked up and designed into clinically effective therapies that are nowadays mainstay therapies for lower urinary tract dysfunction.

Written informed consent for X-ray images from patients was deemed not required by the Radboud University Nijmegen Medical Center for this study because patient data were de-identified from these X-ray images.

\section{Acknowledgment}

This research did not receive any specific grant from funding agencies in the public, commercial or not-for-profit sectors.

\section{Disclosure}

The authors report no conflicts of interest in this work.

\section{References}

1. de Groat WC, Griffiths D, Yoshimura N. Neural control of the lower urinary tract. Compr Physiol. 2015;5(1):327-396.

2. Andersson KE, Arner A. Urinary bladder contraction and relaxation: physiology and pathophysiology. Physiol Rev. 2004;84(3):935-986.

3. Fowler CJ, Griffiths D, de Groat WC. The neural control of micturition. Nat Rev Neurosci. 2008;9(6):453-466.

4. Wein AJ, Rackley RR. Overactive bladder: a better understanding of pathophysiology, diagnosis and management. J Urol. 2006;175(3 pt 2): $5-10$.

5. Nakipoglu GF, Kaya AZ, Orhan G, et al. Urinary dysfunction in multiple sclerosis. J Clin Neurosci. 2009;16(10):1321-1324.

6. Abrams P, Kelleher CJ, Kerr LA, Rogers RG. Overactive bladder significantly affects quality of life. Am J Manag Care. 2000;6(11 Suppl): S580-S590.

7. Araki I, de Groat WC. Developmental synaptic depression underlying reorganization of visceral reflex pathways in the spinal cord. JNeurosci. 1997;17(21):8402-8407.

8. Drake MJ, Apostolidis A, Cocci A, et al. Neurogenic lower urinary tract dysfunction: clinical management recommendations of the Neurologic Incontinence Committee of the fifth International Consultation on Incontinence 2013. Neurourol Urodyn. 2016;35(6):657-665.

9. Dmochowski RR, Gomelsky A. Update on the treatment of overactive bladder. Curr Opin Urol. 2011;21:286-290.

10. Lucas MG, Bosch RJ, Burkhard FC, et al. EAU guidelines on assessment and nonsurgical management of urinary incontinence. Eur Urol. 2012;62(6):1130-1142.
11. de Groat WC, Griffiths D, Yoshimura N. Neural Control of the Lower Urinary Tract. Compr Physiol. 2015;5(1):327-396

12. Fowler CJ, Griffiths D, de Groat WC. The neural control of micturition. Nat Rev Neurosci. 2008;9(6):453-466

13. Yoshikawa S, Kawamorita N, Oguchi T, et al. Pelvic organ crosssensitization to enhance bladder and urethral pain behaviors in rats with experimental colitis. Neuroscience. 2015;284:422-429.

14. de Groat WC, Tai C. Impact of bioelectronic medicine on the neural regulation of pelvic visceral function. Bioelectron Med. 2015: $25-36$.

15. Gebhart GF. Descending modulation of pain. Neurosci Biobehav Rev. 2004;27(8):729-737.

16. Reynolds WS, Dmochowski R, Wein A, Bruehl S. Does central sensitization help explain idiopathic overactive bladder? Nat Rev Urol. 2016; 13(8):481-491.

17. Winnard LP, Dmitrieva N, Berkley KJ. Cross-organ interactions between reproductive gastrointestinal, and urinary tracts: modulation by estrous stage and involvement of the hypogastric nerve. Am J Physiol Regul Integr Comp Physiol. 2006;291(6):1592-1601.

18. Malykhina AP. Neural mechanisms of pelvic organ cross-sensitization. Neuroscience. 2007;149(3):660-672.

19. Pezzone MA, Liang R, Fraser MO. A model of neural cross-talk and irritation in the pelvis: implications for the overlap of chronic pelvic pain disorders. Gastroenterology. 2005;128(7):1953-1964.

20. Finazzi-Agr E, Petta F, Sciobica F, Pasqualetti P, Musco S, Bove P. Percutaneous tibial nerve stimulation effects on detrusor overactivity incontinence are not due to a placebo effect: a randomized, double-blind, placebo controlled trial. J Urol. 2010;184(5):2001-2006.

21. Peters KM, Carrico DJ, Perez-Marrero RA, et al. Randomized trial of percutaneous tibial nerve stimulation versus sham efficacy in the treatment of overactive bladder syndrome: results from the SUmiT trial. J Urol. 2010;183(4):1438-1443.

22. Zhang F, Zhao S, Shen B, et al. Neural pathways involved in sacral neuromodulation of reflex bladder activity in cats. Am J Physiol Renal Physiol. 2013;304(6):710-717.

23. Wang Y, Hassouna MM. Neuromodulation reduces c-fos gene expression in spinalized rats: a double-blind randomized study. J Urol. 2000;163(6):1966-1970.

24. Xiao Z, Rogers MJ, Shen B, et al. Somatic modulation of spinal reflex bladder activity mediated by nociceptive bladder afferent nerve fibers in cats. Am J Physiol Renal Physiol. 2014;307(6):673-679.

25. Brindley GS. Sacral root and hypogastric plexus stimulators and what these models tell us about autonomic actions of the bladder and urethra. Clin Sci (Lond). 1983;70:41s-44s.

26. Blok BF, Groen J, Bosch JL, Veltman DJ, Lammertsma AA. Different brain effects during chronic and acute sacral neuromodulation in urge incontinent patients with implanted neurostimulators. BJU Int. 2006;98(6):1238-1243

27. Kavia R, Dasgupta R, Critchley H, Fowler C, Griffiths D. A functional magnetic resonance imaging study of the effect of sacral neuromodulation on brain responses in women with Fowler's syndrome. BJU Int. 2010;105(3):366-372.

28. Brindley GS, Polkey CE, Rushton DN, Cardozo L. Sacral anterior root stimulators for bladder control in paraplegia: the first 50 cases. J Neurol Neurosurg Psychiatry. 1986;49(10):1104-1114.

29. Brindley GS, Rushton DN. Long-term follow-up of patients with sacral anterior root stimulator implants. Paraplegia. 1990;28(8): 469-475.

30. Martens FM, den Hollander PP, Snoek GJ, Koldewijn EL, van Kerrebroeck PE, Heesakkers JP. Quality of life in complete spinal cord injury patients with a Brindley bladder stimulator compared to a matched control group. Neurourol Urodyn. 2011;30(4):551-555.

31. van Voskuilen AC, Oerlemans DJ, Weil EH, de Bie RA, van Kerrebroeck PE. Long term results of neuromodulation by sacral nerve stimulation for lower urinary tract symptoms: a retrospective single center study. Eur Urol. 2006;49(2):366-372.

32. Dmochowski RR, Gomelsky A. Update on the treatment of overactive bladder. Curr Opin Urol. 2011;21(4):286-290. 
33. Chancellor MB, Chartier-Kastler EJ. Principles of Sacral Nerve Stimulation (SNS) for the treatment of bladder and urethral sphincter dysfunctions. Neuromodulation. 2000;3(1):16-26.

34. Leng WW, Chancellor MB. How sacral nerve stimulation neuromodulation works. Urol Clin North Am. 2005;32(1):11-18.

35. Spinelli M, Sievert KD. Latest technologic and surgical developments in using InterStim Therapy for sacral neuromodulation: impact on treatment success and safety. Eur Urol. 2008;54(6):1287-1296.

36. Pham K, Guralnick ML, O'Connor RC. Unilateral versus bilateral stage I neuromodulator lead placement for the treatment of refractory voiding dysfunction. Neurourol Urodyn. 2008;27(8):779-781.

37. Scheepens WA, de Bie RA, Weil EH, van Kerrebroeck PE. Unilateral versus bilateral sacral neuromodulation in patients with chronic voiding dysfunction. J Urol. 2002;168(5):2046-2050.

38. Kessler TM, Madersbacher H, Kiss G. Prolonged sacral neuromodulation testing using permanent leads: a more reliable patient selection method? Eur Urol. 2005;47(5):660-665.

39. Leong RK, De Wachter SGG, Nieman FHM, de Bie RA, van Kerrebroeck PEV. PNE versus 1st stage tined lead procedure: a direct comparison to select the most sensitive test method to identify patients suitable for sacral neuromodulation therapy. Neurourol Urodyn. 2011;30(7):1249-1252.

40. van Kerrebroeck PEV, van Voskuilen AC, Heesakkers J, et al. Results of sacral neuromodulation therapy for urinary voiding dysfunction: outcomes of a prospective, worldwide clinical study. J Urol. 2007; 178(5):2029-2034.

41. Siddiqui NY, Wu JM, Amundsen CL. Efficacy and adverse events of sacral nerve stimulation for overactive bladder: a systematic review. Neurourol Urodyn. 2010;29(suppl 1):S18-S23.

42. Siegel SW, Catanzaro F, Dijkema HE, et al. Long-term results of a multicenter study on sacral nerve stimulation for treatment of urinary urge incontinence, urgency-frequency, and retention. Urology. 2000; 56(6 suppl 1):87-91.

43. Siegel S, Noblett K, Mangel J, et al. Three-year follow-up results of a prospective, multicenter study in overactive bladder subjects treated with sacral neuromodulation. Urology. 2016;94:57-63.

44. Vandoninck V, Van Balken MR, Agrò EF, et al. Posterior tibial nerve stimulation in the treatment of urge incontinence. Neurourol Urodyn. 2003;22(1):17-23.

45. Staskin DR, Peters KM, MacDiarmid S, Shore N, de Groat WC. Percutaneous tibial nerve stimulation: a clinically and cost effective addition to the overactive bladder algorithm of care. Curr Urol Rep. 2012;13(5): 327-334.

46. McGuire EJ, Zhang SC, Horwinski ER, Lytton B. Treatment of motor and sensory detrusor instability by electrical stimulation. $J$ Urol. 1983;129(1):78-79.

47. Burton C, Sajja A, Latthe PM. Effectiveness of percutaneous posterior tibial nerve stimulation for overactive bladder: a systematic review and meta-analysis. Neurourol Urodyn. 2012;31(8):1206-1216.

48. Govier FE, Litwiller S, Nitti V, Kreder KJ Jr, Rosenblatt P. Percutaneous afferent neuromodulation for the refractory overactive bladder: results of a multicenter study. J Urol. 2001;165(4):1193-1198.

49. Capitanucci ML, Camanni D, Demelas F, Mosiello G, Zaccara A, De Gennaro M. Long-term efficacy of percutaneous tibial nerve stimulation for different types of lower urinary tract dysfunction in children. J Urol. 2009;182(4 suppl):2056-2061.

50. Finazzi-Agr E, Petta F, Sciobica F, Pasqualetti P, Musco S, Bove P. Percutaneous tibial nerve stimulation effects on detrusor overactivity incontinence are not due to a placebo effect: A randomized, doubleblind, placebo controlled trial. J Urol. 2010;184:2001-2006.

51. Peters K, Carrico D, Burks F. Validation of a sham for percutaneous tibial nerve stimulation (PTNS). Neurourol Urodyn. 2009;28(1): 58-61.

52. Peters KM, MacDiarmid SA, Wooldridge LS, et al. Randomized trial of percutaneous tibial nerve stimulation versus extended-release tolterodine: results from the overactive bladder innovative therapy trial. J Urol. 2011;1055:182.
53. MacDiarmid SA, Peters KM, Shobeiri SA, et al. Long-term durability of percutaneous tibial nerve stimulation for the treatment of overactive bladder. J Urol. 2010;183(1):234-240.

54. van der Pal F, van Balken MR, Heesakkers JP, Debruyne FM, Bemelmans BL. Implant-driven tibial nerve stimulation in the treatment of refractory overactive bladder syndrome: 12-month follow-up. Neuromodulation. 2006;9(2):163-171.

55. Janssen DA, Farag F, Heesakkers JP. Urgent-SQ implant in treatment of overactive bladder syndrome: 9-year follow-up study. Neurourol Urodyn. 2013;32(5):472-475.

56. Martens FM, Heesakkers JP, Rijkhoff NJ. Surgical access for electrical stimulation of the pudendal and dorsal genital nerves in the overactive bladder: a review. $J$ Urol. 2011;186(3):798-804.

57. Martens FM, Heesakkers JP, Rijkhoff NJ. Minimal invasive electrode implantation for conditional stimulation of the dorsal genital nerve in neurogenic detrusor overactivity. Spinal Cord. 2011;49(4):566-572.

58. Goldman HB, Amundsen CL, Mangel J, et al. Dorsal genital nerve stimulation for the treatment of overactive bladder symptoms. Neurourol Urodyn. 2008;27(6):499-503.

59. van Breda HM, Farag FF, Martens FM, Heesakkers JP, Rijkhoff NJ. Subject-controlled, on-demand, dorsal genital nerve stimulation to treat urgency urinary incontinence; a pilot. Front Neurosci. 2016;10:24.

60. Spinelli M, Malaguti S, Giardiello G, Lazzeri M, Tarantola J, Van Den Hombergh U. A new minimally invasive procedure for pudendal nerve stimulation to treat neurogenic bladder: description of the method and preliminary data. Neurourol Urodyn. 2005;24(4):305-309.

61. Juenemann KP, Lue TF, Schmidt RA, Tanagho EA. Clinical significance of sacral and pudendal nerve anatomy. J Urol. 1988;139(1):74-80.

62. Groen J, Amiel C, Bosch JL. Chronic pudendal nerve neuromodulation in women with idiopathic refractory detrusor overactivity incontinence: results of a pilot study with a novel minimally invasive implantable mini-stimulator. Neurourol Urodyn. 2005;24(3):226-230.

63. Peters KM, Feber KM, Bennett RC. Sacral versus pudendal nerve stimulation for voiding dysfunction: a prospective, single-blinded, randomized, crossover trial. Neurourol Urodyn. 2005;24(7):643-647.

64. Wang S, Zhang S, Zhao L. Long-term efficacy of electrical pudendal nerve stimulation for urgency-frequency syndrome in women. Int Urogynecol J. 2014;25(3):397-402.

65. Fjorback MV, Van Rey FS, Rijkhoff NJ, Nøhr M, Petersen T, Heesakkers JP. Electrical stimulation of sacral dermatomes in multiple sclerosis patients with neurogenic detrusor overactivity. Neurourol Urodyn. 2007;26(4):525-530.

66. Gross T, Schneider MP, Bachmann LM, et al. Transcutaneous electrical nerve stimulation for treating neurogenic lower urinary tract dysfunction: a systematic review. Eur Urol. 2016;69(6):1102-1111.

67. Guo ZF, Liu Y, Hu GH, Liu H, Xu YF. Transcutaneous electrical nerve stimulation in the treatment of patients with poststroke urinary incontinence. Clin Interv Aging. 2014;9:851-856.

68. Opisso E, Borau A, Rijkhoff NJ. Subject-controlled stimulation of dorsal genital nerve to treat neurogenic detrusor overactivity at home. Neurourol Urodyn. 2013;32(7):1004-1009.

69. Farag FF, Martens FM, Rijkhoff NJ, Heesakkers JP. Dorsal genital nerve stimulation in patients with detrusor overactivity: a systematic review. Curr Urol Rep. 2012;13(5):385-388.

70. Barker AT, Freeston IL, Jalinous R, Jarratt JA. Magnetic stimulation of the human brain and peripheral nervous system: an introduction and the results of an clinical initial evaluation. Neurosurgery. 1987;20(1): 100 .

71. Fujishiro T, Enomoto H, Ugawa Y, Takahashi S, Ueno S, Kitamura T. Magnetic stimulation of the sacral roots for the treatment of stress incontinence: an investigational study and placebo controlled trial. J Urol. 2000;164(4):1277-1279.

72. Lo TS, Tseng LH, Lin YH, Liang CC, Lu CY, Pue LB. Effect of extracorporeal magnetic energy stimulation on bothersome lower urinary tract symptoms and quality of life in female patients with stress urinary incontinence and overactive bladder. J Obstet Gynaecol Res. 2013;39(11):1526-1532. 


\section{Publish your work in this journal}

Medical Devices: Evidence and Research is an international, peerreviewed, open access journal that focuses on the evidence, technology, research, and expert opinion supporting the use and application of medical devices in the diagnosis, monitoring, treatment and management of clinical conditions and physiological processes. The identification of novel devices and optimal use of existing devices which will lead to improved clinical outcomes and more effective patient management and safety is a key feature. The manuscript management system is completely online and includes a quick and fair peer-review system. Visit http://www. dovepress.com/testimonials.php to read real quotes from authors.

Submit your manuscript here: https://www.dovepress.com/medical-devices-evidence-and-research-journal 